\title{
Light Lepton Number Violating Sneutrinos and the Baryon Number of the Universe
}

\author{
H.V. Klapdor-Kleingrothaus ${ }^{\dagger}$, St. Kolb ${ }^{\dagger \ddagger}$ and V.A. Kuzmin ${ }^{\ddagger}$ \\ † Max-Planck-Institut für Kernphysik, P.O. 1039 80, D-69029 Heidelberg, \\ Germany \\ $\ddagger$ Institute for Nuclear Research of the Russian Academy of Sciences, 60th October \\ Anniversary Prospect 7a, 117321 Moscow, Russia
}

\begin{abstract}
Recent results of neutrino oscillation experiments point to a non-vanishing neutrino mass. Neutrino mass models favour Majorana-type neutrinos. In such circumstances it is natural that the supersymmetric counterpart of the neutrino, the sneutrino, bears also lepton number violating properties. If the amount of lepton number violation is large enough the sneutrino may be the Cold Dark Matter in the universe. On the other hand, the fact that the universe exhibits an asymmetry in the baryon and antibaryon numbers poses constraints on the extent of lepton number violation in the light sneutrino sector if the electroweak phase transition is second or weak first order. From the requirement that the Baryon Asymmetry of the Universe should not be washed out by sneutrino induced lepton number violating interactions and sphalerons below the critical temperature of the electroweak phase transition we find that the mass-splitting of the light sneutrino mass states is compatible with the sneutrino Cold Dark Matter hypothesis only for heavy gauginos $M_{1}, M_{2} \gtrsim 500 \mathrm{GeV}$ and opposite sign gaugino mass parameters.
\end{abstract}

\section{Introduction}

There are hints from neutrino oscillation experiments that the neutrino is massive ([1] and refs. therein. For a recent overview see e.g. [2]). In most neutrino mass models the neutrino is of Majorana-type, i.e. it violates lepton number $L$. If this is indeed the case the next generation of experiments searching for neutrinoless double beta $(0 \nu \beta \beta)$ decay, which are the only experiments capable of deciding on the nature of the neutrino, possibly will be able to indeed observe a $0 \nu \beta \beta$-decay signal (for a recent overview see $e . g$. [3]).

On the other hand, it has been shown in [4] that if the neutrino is a massive Majorana field the low energy effective theory of the supersymmetric extension of the Standard Model (for a phenomenological overview see e.g. [5]) will contain mass terms for the sneutrino which violate $L$ too, regardless of the mechanism which is 
responsible for the generation of sneutrino masses in the unbroken theory. In [6] a model containing heavy $S U(2)$ singlet sneutrino fields and $L$-violating mass terms involving these fields has been examined (such models have been considered previously in connection with the generation of the Baryon Asymmetry of the Universe (BAU) at some high temperature, see e.g. [0]). In both cases below the electroweak symmetry breaking scale the weak states $\tilde{\nu}, \tilde{\nu}^{*}$ are no longer mass states and the resulting mass states violate $L$, exhibit a mass-splitting and give rise to $L$-violating processes which have been analyzed e.g. for the Next Linear Collider in [8] and for $0 \nu \beta \beta$ decay in [9].

It has been pointed out in [10] that in a scenario where the light sneutrino mass states exhibit a mass-splitting the lightest sneutrino could account for the Cold Dark Matter (CDM) in the universe. This is due to the fact that the sneutrino mass states couple "off-diagonally" to the $Z^{0}$ on grounds of Bose statistics and angular momentum conservation so that the arguments excluding ordinary sneutrinos from constituting a substantial fraction of the CDM are not valid in the case of light sneutrinos exhibiting a mass difference. However, it has been shown in [10] that the mass difference should be of order $\mathcal{O}$ (few $\mathrm{GeV}$ ).

On the other hand sneutrino mediated $L$-violating reactions, like any other $L$ violating process, may be dangerous for the BAU due to sphalerons [11. The constraints on the $L$-violating sneutrino properties stemming from the requirement that a BAU generated at some early epoch in the evolution of the universe should not be destroyed by sneutrino-induced interactions during a later epoch are the subject of this note.

\section{Lepton number violation in the sneutrino sector}

In the following the discussion will be restricted to the one-generation case. We plan to investigate the impact of possible $C P$ violation in a multi-generation scheme for the light sneutrino sector elsewhere. It has been pointed out in 4 that the low energy (i.e below the scale where the $S U(2)_{L} \times U(1)_{Y}$ gauge group is broken into $\left.U(1)_{e m}\right)$ sneutrino mass terms can be written as

$$
\mathcal{L}_{\text {mass }}^{\text {eff }}=-\frac{1}{2}\left(\tilde{m}_{D}^{2} \tilde{\nu}_{L} \tilde{\nu}_{L}^{*}+\tilde{m}_{M}^{2} \tilde{\nu}_{L} \tilde{\nu}_{L}+\text { h.c. }\right) .
$$

Here $\tilde{m}_{D}^{2}$ contains as usual contributions from $V_{\text {soft }}$ and from the D-term, whereas $\tilde{m}_{M}^{2}$ violates $L$ explicitly and may have its origin at some high energy scale. Furthermore, loops containing Majorana neutrinos and neutralinos induce contributions to $\tilde{m}_{M}^{2}$ radiatively. Expression (1) is valid if no right-handed sneutrino sector is present in the theory and independent of the mechanism which generates the $L$-violating sneutrino mass. Writing $\tilde{\nu}_{L}=1 / \sqrt{2}\left(\tilde{\nu}_{1}+i \tilde{\nu}_{2}\right)$ where $\tilde{\nu}_{1,2}$ are real, the resulting mass 
states are simply $\tilde{\nu}_{1,2}$ with masses $m_{1 / 2}^{2}=\tilde{m}_{D}^{2} \pm \tilde{m}_{M}^{2}$ and the mass difference is $\Delta m^{2}=2 \tilde{m}_{M}^{2}$.

If a right-handed sector is included the sneutrino mass terms after electroweak symmetry breaking without an explicit $L$-violating mass term for the $S U(2)$ doublet sneutrinos are [6]

$$
\mathcal{L}_{\text {mass }}=-\frac{1}{2}\left(\phi_{1}, \phi_{2}\right)\left(\begin{array}{cc}
\mathcal{M}_{+}^{2} & 0 \\
0 & \mathcal{M}_{-}^{2}
\end{array}\right)\left(\begin{array}{l}
\phi_{1} \\
\phi_{2}
\end{array}\right)
$$

where $\phi=\left(\tilde{\nu}_{i}, \tilde{N}_{i}\right)^{T}$ and $\tilde{N}=\left(\tilde{N}_{1}+i \tilde{N}_{2}\right) / \sqrt{2}$ is the $S U(2)_{L}$ singlet field. The matrices $\mathcal{M}_{ \pm}^{2}$ are defined as

$$
\mathcal{M}_{ \pm}^{2}=\left(\begin{array}{cc}
m_{\tilde{L}}^{2}+\frac{1}{2} m_{Z}^{2} \cos 2 \beta+m_{D}^{2} & m_{D}\left(A_{\nu}-\mu \cot \beta \pm M\right) \\
m_{D}\left(A_{\nu}-\mu \cot \beta \pm M\right) & M^{2}+m_{D}^{2}+m_{\tilde{N}}^{2} \pm 2 B_{N} M
\end{array}\right) .
$$

The parameters $m_{\tilde{N}}^{2}$ and $A_{\nu}$ are contained in $V_{\text {soft }}$ in analogy to the charged sfermion sectors. Furthermore $V_{\text {soft }}$ contains a term $M B_{N} \tilde{N} \tilde{N}+$ h.c. which violates $L$. The remaining entries of the mass matrix (2) are the $F$-terms stemming from the superpotential which in comparison to the MSSM contains an additional term $M \hat{N} \hat{N}$. The Dirac neutrino mass is $m_{D}=\lambda v_{2}, \lambda$ being a Yukawa coupling, $v_{i} / \sqrt{2}$ is the vacuum expectation value of the neutral component of the $S U(2)$ doublet Higgs $H_{i}$, and $\tan \beta=v_{2} / v_{1}$. It is natural that $M \gg m_{Z}$ since $m_{\nu} \approx m_{D}^{2} / M, \mu, A_{\mu}, m_{\tilde{L}} \sim \mathcal{O}\left(m_{Z}\right)$ since sfermions with nontrivial $S U(2)_{L} \times U(1)_{Y}$ transformation properties should not be much heavier than $100 \mathrm{GeV}-1 \mathrm{TeV}$, whereas $B_{N}, m_{\tilde{N}}$ may a priori be much bigger since they pertain to the $S U(2)_{L} \times U(1)_{Y}$ singlet field $\tilde{N}, c f$. the discussion in $[6]$.

For the matrix (2) the mass states read

$$
\begin{gathered}
\xi_{1,2}^{l}=\cos \Theta_{ \pm} \tilde{\nu}_{1,2}+\sin \Theta_{ \pm} \tilde{N}_{1,2} \\
\xi_{1,2}^{h}=-\sin \Theta_{ \pm} \tilde{\nu}_{1,2}+\cos \Theta_{ \pm} \tilde{N}_{1,2}
\end{gathered}
$$

where the angles $\Theta_{ \pm}$diagonalize $\mathcal{M}_{ \pm}^{2}$ and the indices $l, h$ refer to light and heavy sneutrino mass states. In leading order in $1 / M$ the mass difference of the light sneutrino states is

$$
\Delta m^{2}=m_{\xi_{2}^{l}}^{2}-m_{\xi_{1}^{l}}^{2}=4 \frac{m_{D}^{2}}{M}\left(A_{\nu}-\mu \cot \beta-B_{N}\right)
$$

in accordance with [6].

In the following it will not be distinguished further between the models eq. (1) and eq. (2) and the light sneutrino mass states will be denoted by $\xi_{1,2}^{l}$. Note that the mass difference $\Delta m$ of the light states is related to $\Delta m^{2}$ by

$$
\Delta m=m_{\xi_{2}^{l}}-m_{\xi_{1}^{l}}=\Delta m^{2} /\left(m_{\xi_{1}^{l}}+m_{\xi_{2}^{l}}\right) .
$$




\section{Baryon number depletion by sneutrinos below the critical temper- ature}

In both scenarios eqs. (11), (2) sneutrinos give rise to $L$-violating processes, e.g. sneutrino decays or $2 \leftrightarrow 2$ scatterings. These scatterings have the potential to erase an asymmetry in the number of baryons and antibaryons in the early universe. Such an asymmetry has to be generated somewhen during the evolution of the universe in order to explain the absence of antimatter in the universe observed (for an overview see e.g. 12]). In the following it is assumed that at some high temperature above the electroweak symmetry breaking scale the BAU has been generated by some mechanism in the right amount. One example of such a mechanism is the decay of heavy right-handed neutrinos and/or sneutrinos which incorporates the three basic conditions for the generation of the BAU: baryon or lepton number violation, $C P$-violation and out of thermal equilibrium circumstances. All three conditions may be satisfied by the decay of $S U(2)_{L}$ singlet sneutrinos, in particular the out of equilibrium condition requires them to be very massive $\left(\mathcal{O}\left(10^{10} \mathrm{GeV}\right)\right)$ in accordance with the considerations mentioned above $\left(M \gg \mathcal{O}\left(m_{Z}\right)\right)$.

However, at temperatures below the electroweak symmetry breaking scale $T \sim$ $\mathcal{O}(100 \mathrm{GeV})$ the light sneutrino states violate $L$ too and sneutrino interactions may bring the distributions of leptons and antileptons into equilibrium. An estimation of interactions induced by Majorana neutrinos below $T_{C}$ has been given in [13. The approximate criterium for equilibration is that the rate of the process in question should be bigger than the expansion rate of the universe $H(T)=1.7 \sqrt{g_{*}} T^{2} / M_{P l}$ (in a radiation dominated universe) where $g_{*} \approx 200$ in the MSSM and $M_{P l} \approx 10^{19} \mathrm{GeV}$.

As long as sphaleron-mediated processes are operative the lepton and baryon numbers are both proportional to the combination $B-L$ 11. As a consequence the asymmetry in baryons vanishes if the asymmetry in leptons is somehow erased. Hence, if there is a temperature range between the critical temperature of the $S U(2)_{L} \times U(1)_{Y} \rightarrow U(1)_{\text {em }}$ phase transition and the temperature $T_{\text {out }}$ at which sphaleron-mediated processes drop out of equilibrium (i.e. $\Gamma_{S p h}\left(T_{\text {out }}\right)<H\left(T_{\text {out }}\right)$ where $\Gamma_{S p h}(T)$ is the sphaleron rate) so that

$$
T_{\text {out }}<T<T_{C}
$$

then $L$-violating interactions are potentially dangerous for the BAU. The presence of a temperature range satisfying (7) is not possible in models where the BAU is generated during the electroweak phase transition itself (Electroweak Baryogenesis) since in such models the sphaleron-mediated processes must be switched off immediately below $T_{C}$. Therefore any constraints on the $L$-violating properties of light sneutrinos do a priori not hold in the context of Electroweak Baryogenesis or any other model where sphaleron-mediated processes are out of equilibrium immediately after the electroweak phase transition. 
In order to estimate the temperature range (7) the following assumptions are made: the evolution of the vacuum expectation value (vev) of the Higgs fields $\langle v(T)\rangle$ is described by

$$
\langle v(T)\rangle=\langle v(0)\rangle\left(1-T^{2} / T_{C}^{2}\right)^{1 / 2},\langle v(0)\rangle=246 G e V .
$$

This behaviour of the vev is valid for a second order phase transition and approximately valid for a weak first order phase transition. The exact behaviour of $\langle v(T)\rangle$ depends on the SUSY parameters, but for our phenomenological purposes we will satisfy ourselves with eq. (8). Lattice simulations suggest that $T_{C} \approx 150 \mathrm{GeV}$ for a Higgs mass of $70 \mathrm{GeV}$ 14 and that it should be higher for larger values of the Higgs mass, but in order to keep the results general we will vary $T_{C}$ freely between $50 \mathrm{GeV}$ and $250 \mathrm{GeV}$. In models of electroweak baryogenesis sphalerons have to drop out of equilibrium immediately after the phase transition which translates into the condition 15] $\left\langle v\left(T=T_{C}\right)\right\rangle / T_{C}>1$ in contrast to (8), see the comment above.

The sphaleron rate below $T_{C}$ is described by (15 and refs. therein)

$$
\Gamma_{S p h} \approx 2.8 \cdot 10^{5} T^{4} \kappa\left(\frac{\alpha_{W}}{4 \pi}\right)^{4}\left(\frac{2 m_{W}(T)}{\alpha_{W} T}\right)^{7} \exp \left(-\frac{E_{s p}(T)}{T}\right)
$$

where

$$
m_{W}(T)=\frac{1}{2} g_{2}\langle v(T)\rangle,
$$

the free energy of the sphaleron configuration is given by

$$
E_{S p h}(T)=\frac{2 m_{W}(T)}{\alpha_{W}} B\left(\frac{m_{H}}{m_{W}}\right),
$$

$B(0)=1.52, B(\infty)=2.72$ and $\kappa=\exp (-3.6)$ [16]. In figure 1 the temperature range $T_{C}-T_{\text {out }}$ is plotted as a function of $T_{C}$. It becomes smaller than one for small values of $T_{C} \lesssim 100 \mathrm{GeV}$ but is of order $\mathcal{O}(10 \mathrm{GeV})$ for $T_{C} \sim 200 \mathrm{GeV}$.

For $L$-violating sneutrino decays and $L$-violating sneutrino mediated scatterings the damping of the preexisting Lepton number (per comoving volume) $L_{i}$ is described by the Boltzmann equation for the evolution of $L$ and is given by (see e.g. [12])

$$
\left.L(z)=L_{i} \exp \left[-\int_{z_{c}}^{z_{\text {out }}} d z^{\prime} z^{\prime}\left[g_{*} \frac{n_{\tilde{\nu}}}{s} \Gamma_{D}\left(z^{\prime}\right)+n_{\gamma}\langle\sigma|v|\rangle\right)\right] / H\left(T=m_{\tilde{\nu}}\right)\right]
$$

where $z=m_{\tilde{\nu}} / T, \Gamma_{D}$ is the $L$-violating sneutrino decay width, $\langle\sigma|v|\rangle$ is the thermally averaged cross-section of sneutrino mediated $L$-violating scatterings and $n_{i}$ is the number density of particle $i$ (in the scattering term the number density of the initial state particles has been assumed to be the photon number density). In order to 


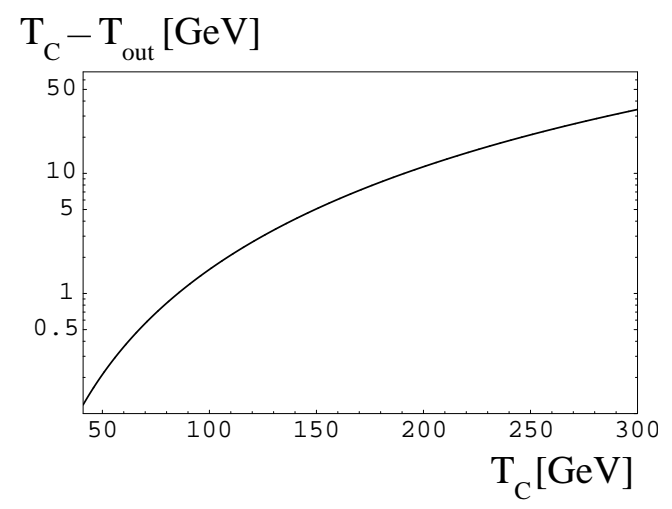

Figure 1: The difference of the critical temperature $T_{C}$ and the sphaleron freezing out temperature $T_{\text {out }}$ determined by $\Gamma_{\text {sph }}\left(T_{\text {out }}\right)=H\left(T_{\text {out }}\right)$ in dependence of $T_{C}$.

derive bounds on $L$ violation we will allow that during the epoch $T_{\text {out }}<T<T_{C} L$ may be depleted by a factor $k<1$, that is we assume the preliminary $L$ density to be $n_{L} / n_{\gamma} \approx 10^{-10} / k$.

\section{Constraints on the sneutrino mass-splitting}

In the following two different cases will be distinguished: I) at least one gaugino (see below) or slepton other than the sneutrino are lighter than $T_{C}$, that is the number density of particles satisfying this condition is approximately the photon number density; II) all gauginos and sleptons besides the sneutrino are (substantially) heavier than $T_{C}$ so that the number density of these particles is exponentially suppressed and one sneutrino is the lightest supersymmetric particle (LSP). The discussion will be focused on the case of sneutrino CDM, that is on a mass difference of order $5 \mathrm{GeV}$ and a sneutrino LSP mass of $m_{L S P} \sim 70 \mathrm{GeV}$ 10.

I) In order to keep the results as general as possible only scatterings will be taken into account in what follows since these depend to a smaller extent on the exact mass relations of the particles involved than $L$-violating decays do. Then relevant processes capable of depleting $L$ are $2 \leftrightarrow 2$ scatterings as for example scattering of neutralinos or charginos into leptons mediated by $L$-violating sneutrinos (see figure 2)

$$
\chi_{i} \chi_{j} \longleftrightarrow l_{l} l_{m} \quad, \quad \chi_{i} l_{l} \longleftrightarrow \bar{\chi}_{j} \bar{l}_{m}
$$

where $\chi_{k}$ represents any of the chargino or neutralino mass fields present in the plasma at $T<T_{C}$ and $l_{n}$ is a charged or uncharged lepton. In the one generation case $l=m$. However, since the entries of the neutralino mixing matrix connecting gauginos with higgsinos are proportional to the vev this mixing will be suppressed by 


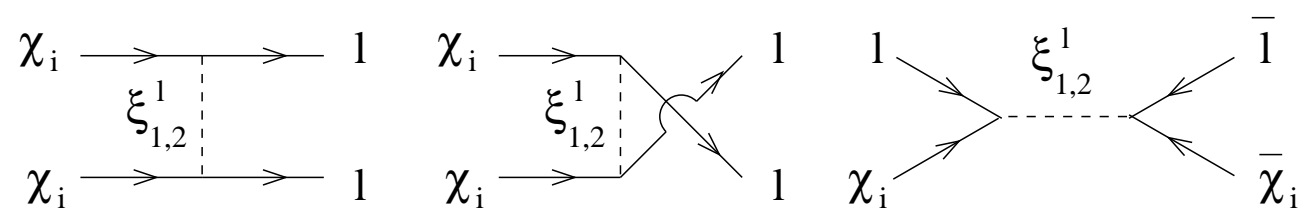

Figure 2: Graphs for $2 \leftrightarrow 2$ scatterings of gauginos $\chi_{i}$ and leptons $l$ mediated by the light sneutrino states $\xi_{1,2}^{l}$.

$\left\langle v\left(T=T_{\text {out }}\right)\right\rangle /\langle v(T=0)\rangle$ and therefore $\chi$ will be assumed to be $\tilde{B}$ with associated mass $M_{1}$ and $\tilde{W}^{3}, \tilde{W}^{ \pm}$with associated mass $M_{2}$.

The following discussion will be restricted to the process eq. (13), but for other examples as $\tilde{l}^{ \pm} \tilde{l}^{ \pm} \longleftrightarrow W^{ \pm} W^{ \pm}$the same arguments hold. The constraint on the mass-splitting follows from the relation

$$
\int_{z_{C}}^{z_{\text {out }}} d z^{\prime} z^{\prime} \frac{1}{H\left(m_{\tilde{\nu}}\right)} n_{\gamma}\langle\sigma|v|\rangle>\ln k
$$

The zero-temperature cross-section of this reaction is proportional to $\left(\Delta m^{2}\right)^{2}$ since 44

$$
\langle 0|T[\tilde{\nu}(x) \tilde{\nu}(y)]| 0\rangle=\Delta m^{2} \frac{i}{2} \int \frac{d^{4} k}{(2 \pi)^{4}} \frac{e^{-i k(x-y)}}{\left(m_{\xi_{1}^{l}}^{2}-k^{2}\right)\left(m_{\xi_{2}^{l}}^{2}-k^{2}\right)+i \epsilon}
$$

where for model eq. (2) the contributions of the heavy states have been neglected. Therefore the final bound on $\Delta m$ depends only weakly on the precise value of $\langle\sigma|v|\rangle$ (and on $k$ ) and for our purposes it is sufficient to approximate the thermally averaged scattering cross section by

$$
\langle\sigma|v|\rangle \approx\left(\Delta m^{2}(T)\right)^{2} \frac{\alpha_{W}^{2} T^{2}}{\left(T^{2}+m_{\tilde{\nu}}^{2}\right)^{4}}
$$

where $\alpha_{W}$ is the weak coupling constant and for non-vanishing temperatures and a second order phase transition (see eq. 6)

$$
\frac{\Delta m(T)}{\Delta m(T=0)}=\frac{\Delta m^{2}(T)}{\Delta m^{2}(T=0)}=\frac{\langle v(T)\rangle^{2}}{\langle v(T=0)\rangle^{2}}=\left(1-\frac{T^{2}}{T_{C}^{2}}\right) .
$$

The full zero temperature cross section can be found in [8].

The results, which hold for any generation, are plotted in figure 3 for several values for the sneutrino mass and $k$ in dependence of $T_{C}$ which has been varied freely between $50 \mathrm{GeV}$ and $250 \mathrm{GeV}$. For example for $T_{C} \approx 150 \mathrm{GeV}$ the limits range from 

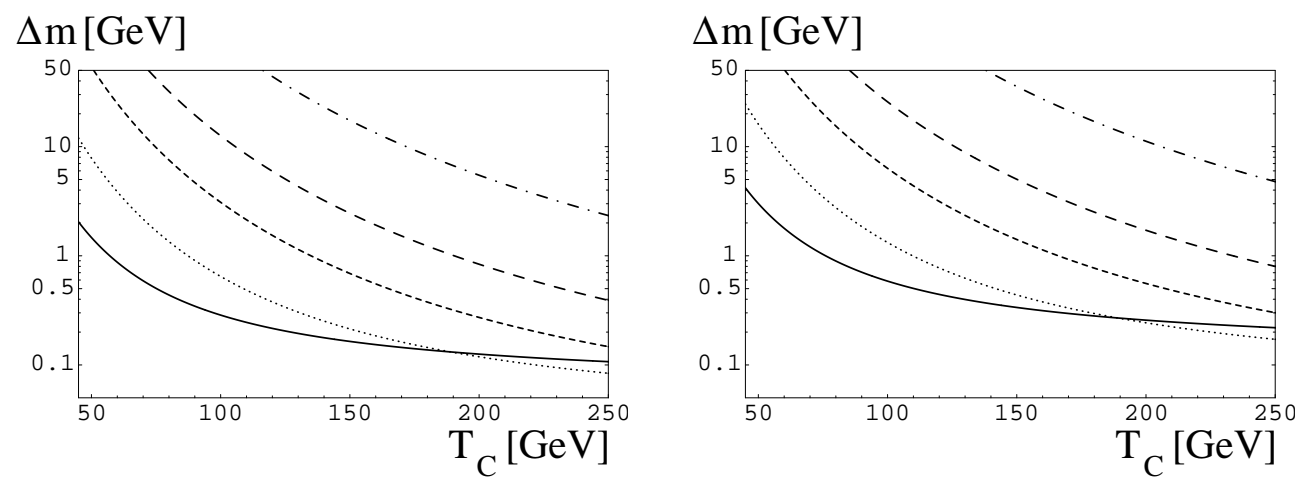

Figure 3: The limits on the zero temperature light sneutrino mass-splitting splitting $\Delta m(T=0)$ in case $\mathbf{I})$ (see text) in dependence of the critical temperature $T_{C}$ from eq. (14) (the regions above the curves are excluded) for $k=0.3$ (left) and $k=0.01$ (right) and for different values of the sneutrino mass: $m_{\xi^{l}}=70 \mathrm{GeV}$ (solid curve), $m_{\xi^{l}}=150 \mathrm{GeV}$ (dotted curve), $m_{\xi^{l}}=300 \mathrm{GeV}$ (short-dashed curve), $m_{\xi^{l}}=500 \mathrm{GeV}$ (long-dashed curve), $m_{\xi^{l}}=1 T e V$ (dot-dashed curve).

$\Delta m \lesssim \mathcal{O}($ few $100 \mathrm{MeV})$ to $\Delta m \lesssim \mathcal{O}($ few $\mathrm{GeV})$ for the chosen sneutrino masses. The limit on the mass-splitting becomes less stringent for smaller values of $T_{C}$ (since the temperature range (7) becomes smaller) and for larger values of $m_{\tilde{\nu}}$. For small values of $T_{C}$ and large values of $m_{\tilde{\nu}}$ the mass-splitting is basically not constrained.

II) In the case that all gauginos and sleptons but the sneutrinos have already decayed away at temperatures above $T_{C}$ the processes depleting $L$ are $2 \leftrightarrow 2$ scatterings of sneutrinos into neutrinos and $L$-violating decays of non-LSP sneutrinos into the LSP sneutrino $\xi^{h} \rightarrow \xi^{L S P} \nu \nu$ mediated by neutralinos.

The cross-section for gaugino-mediated $L$-violating scattering of sneutrinos into neutrinos can be approximated by (compare with [10])

$$
\langle\sigma|v|\rangle \approx \frac{\left(\Delta m^{2}(T)\right)^{2}}{T^{4}}\left(\frac{\alpha_{Y} M_{1}}{T^{2}+M_{1}^{2}}+\frac{\alpha_{W} M_{2}}{T^{2}+M_{2}^{2}}\right)^{2}
$$

where $\alpha_{Y}$ is the coupling associated with $U(1)_{Y}$. This cross-section depends sensitively on the relation $M_{2} / M_{1}$. In figure 4 the bounds on the $\Delta m$ are shown for several values of $M_{1}$ and for the cases of $M_{2} / M_{1}$ considered in [10, 18] which have been shown to be compatible with the sneutrino CDM hypothesis as long as $M_{1} \gtrsim 200 \mathrm{GeV}$ (see next section).

The resulting limits are more stringent than the bounds in I) if $M_{1}$ and $M_{2}$ have the same sign. This is simply due to the propagator structure of the two 

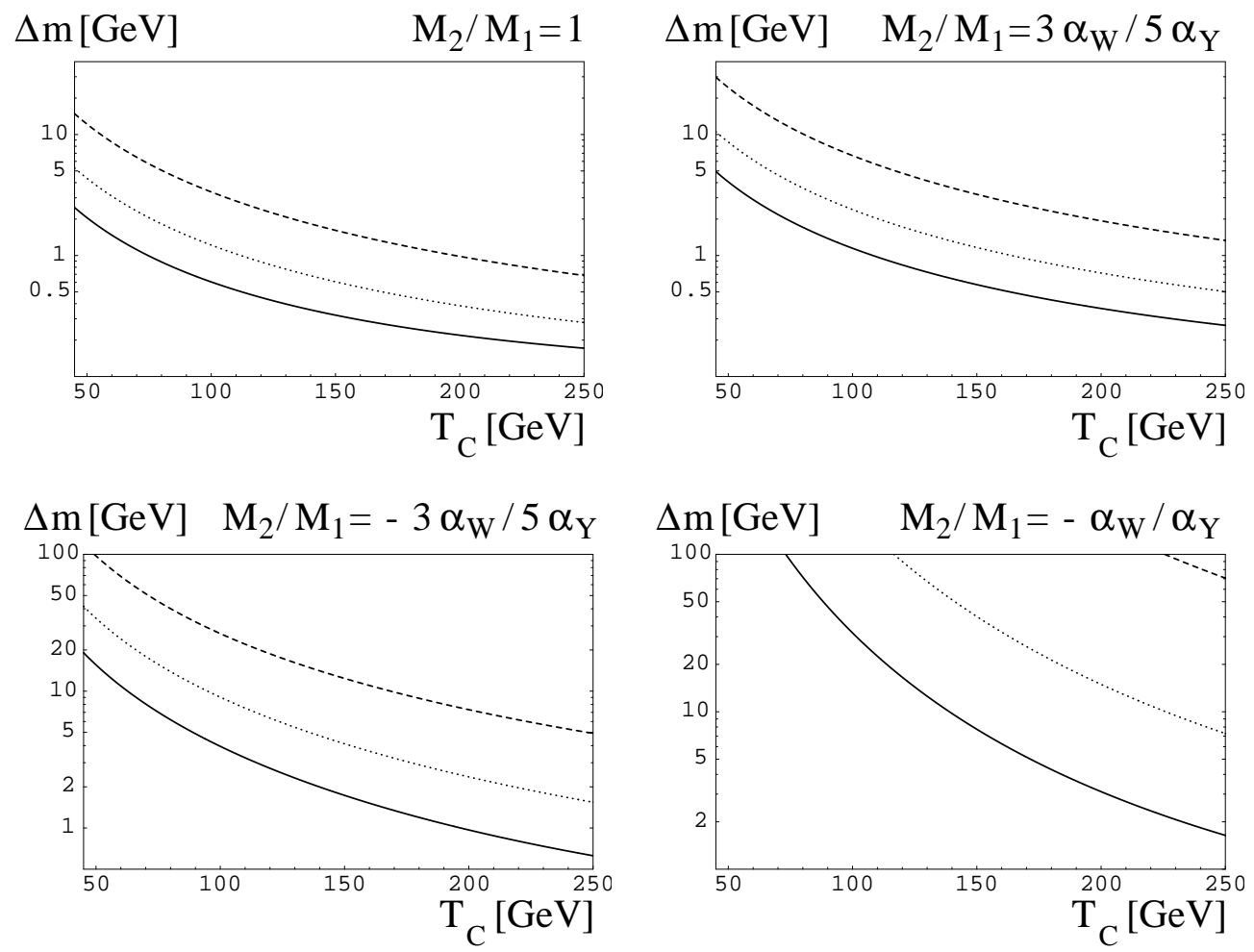

Figure 4: The limits on $\Delta m(T=0)$ from eqs. (18), (14) in case II) (see text) for several values of the ratio $M_{2} / M_{1}$ in dependence on the critical temperature and for a depletion factor $k=1 / 3$. The regions above the lines are excluded. The limits correspond to the parameters $M_{1}=300 \mathrm{GeV}$ (solid line), $M_{1}=500 \mathrm{GeV}$ (dotted line) and $M_{1}=1 T e V$ (dashed line).

different scattering processes. Even for very large gaugino masses of order $\mathcal{O}(\mathrm{TeV})$ the mass-splitting $\Delta m(T=0)$ is only of order $\mathcal{O}($ few $G e V)$ for plausible values of $T_{C}$. This statement is also valid in the limit $M_{2} \rightarrow 0$. On the other hand, if $M_{1}$ and $M_{2}$ are of opposite sign there is a partial cancellation of the contributions and the resulting limits on $\Delta m(T=0)$ become weaker. In particular for $T_{C} \approx 200 \mathrm{GeV}$ for $M_{2} / M_{1}=-3 \alpha_{W} / 5 \alpha_{Y}\left(-\alpha_{W} / \alpha_{Y}\right)$ a mass difference $\Delta m \sim \mathcal{O}($ few GeV $)$ is allowed if $M_{1} \approx 500 \mathrm{GeV}(300 \mathrm{GeV})$.

The decays of non-LSP sneutrinos into the LSP sneutrino have been discussed in detail in [18]. Neglecting gaugino mixing the zero-temperature decay width of the 
$L$-violating mode to leading order in the parameter $\Delta m^{2}$ is

$$
\Gamma_{\amalg} \approx \frac{\alpha_{W}^{2}}{16 \pi} \frac{\left(\Delta m^{2}(T)\right)^{5}}{\left(m_{\chi}^{2}-m_{+}^{2}\right)^{4} \sqrt{m_{+}^{2}}} \sim\left(\Delta m^{2}(T=0)\right)^{5}\left(1-\frac{T^{2}}{T_{C}^{2}}\right)^{5}
$$

where $m_{+}^{2}=m_{\xi L S P}^{2}+m_{\xi^{h}}^{2}$. This expression does not result into a constraint when inserted into eq. (12) due to its dependence on the fifth power of the suppression factor $\left(1-T^{2} / T_{C}^{2}\right)$ : e.g. for $T_{C} \lesssim 250 \mathrm{GeV}$ the constraint on the mass-splitting is $\Delta m \lesssim \mathcal{O}(100 \mathrm{GeV})$ and for smaller values of $T_{C}$ becomes even less stringent.

\section{Comparison with low-energy constraints and implications for sneu- trino Cold Dark Matter}

In [4, 6] it has been shown that $L$ violating sneutrinos give rise to loops containing neutralinos and (light) sneutrinos which contribute to the (Majorana) mass of the neutrino. In [9] a scan over a wide range of the SUSY parameter has been carried out and "average" upper limits

$$
\Delta m(e)<8 M e V, \Delta m(\mu)<6 G e V
$$

have been deduced (for neutrino mass limits $m(e)<15 \mathrm{eV}$ and $m(\mu)<170 \mathrm{keV}$ ) while the third generation mass-splitting remains virtually unconstrained. The current bound on the effective Majorana neutrino mass $\left\langle m_{e e}\right\rangle=\sum_{i}^{\prime} U_{e i}^{2} m_{i}<0.36 \mathrm{eV}$ (90\% c.l.) from the Heidelberg-Moscow experiment [17] yields in the case $U_{e i} \approx \delta_{e i}$ the relation $\Delta m(e)<13 \mathrm{keV}$ for an average sneutrino mass of $100 \mathrm{GeV}$. Direct contributions to $0 \nu \beta \beta$ have been considered in [9] and the resulting limits on $\Delta m(e)$ are less stringent. Therefore the limits from baryogenesis are considerably less severe than the ones from neutrino masses in the first generation case for plausible ranges of $T_{C}$ and superpartner masses. For the second generation the limit is comparable or slightly more stringent for values of $T_{C}$ not much smaller than $150 \mathrm{GeV}$ and superpartner masses not bigger than $500 \mathrm{GeV}$. In the third generation case for plausible values of $T_{C}$ baryogenesis yields more stringent limits than neutrino masses do.

The constraints on the sneutrino mass-splitting are especially interesting for the hypothesis of sneutrino CDM. In [10] it has been shown that a sneutrino with mass of around $70 \mathrm{GeV}$ may be a viable CDM candidate if the mass-splitting exceeds about $5 G e V$ (so that $Z^{0}$-mediated $s$-channel coannihilation of the LSP sneutrino with its heavier partner is sufficiently suppressed) and $M_{1}$ is bigger than about $200 \mathrm{GeV}$ (so that $L$-conserving LSP-sneutrino pair annihilation is sufficiently suppressed). There-

fore the low energy limits eq. (20) imply that the light third generation sneutrino is a viable CDM candidate. On the other hand, in case I) discussed in the last chapter the results displayed in figure 3 (solid line) imply that sneutrino CDM is firmly ruled out for plausible values of the critical temperature $T_{C} \gtrsim 150 \mathrm{GeV}$. 
The same conclusion is valid in scenario II) for plausible values of $T_{C}$ if $M_{1}$ and $M_{2}$ bear the same sign as long as the gauginos are not exceptionally heavy $\left(M_{1} \gtrsim 1 T e V\right)$. However, in case II) the bound on the sneutrino mass-splitting could become sufficiently large to allow for sneutrino CDM if $M_{1}$ and $M_{2}$ are of opposite sign. For plausible values of $T_{C} \gtrsim 150 \mathrm{GeV}$ the ratio $M_{2} / M_{1}=-3 \alpha_{W} / 5 \alpha_{Y}$ $\left(M_{2} / M_{1}=-\alpha_{W} / \alpha_{Y}\right)$ allows for a sneutrino mass-splitting being large enough to be compatible with the sneutrino CDM hypothesis as long as $M_{1}$ is bigger than about $500 \mathrm{GeV}(300 \mathrm{GeV})$. In particular, this statement is compatible with the lower limit on $M_{1}$ from the requirement that the sneutrino relic abundance should be sufficiently high to account for the CDM.

\section{Summary}

In conclusion, in scenarios with $L$ violation in the light sneutrino sector it has to be made sure that sneutrino-induced interactions which take place in the early universe at temperatures below the electroweak symmetry breaking scale do not erase the BAU generated at some higher temperature. This may happen if the electroweak phase transition is second or weakly first order, so that sphaleron-induced processes are still operative at temperatures below the critical temperature $T_{C}$. The $L$-violating interactions considered here result in constraints on the mass-splitting of the light sneutrino mass states which are less stringent than the ones which can be derived from the contribution of $L$-violating sneutrinos to neutrino masses for the first generation. For the second generation the limits are more stringent for plausible values of $T_{C} \gtrsim 150 \mathrm{GeV}$ and for superpartner masses smaller than $500 \mathrm{GeV}$ except for the case of an almost complete cancellation of the bino and wino contributions for a sneutrino LSP of mass $\mathcal{O}(100 \mathrm{GeV})$.

In particular, the constraint on the third generation mass-splitting is of interest for the sneutrino Cold Dark Matter hypothesis. Low energy limits on the sneutrino mass-splitting suggest that the lighter of the third generation sneutrino states with mass of around $70 \mathrm{GeV}$ may serve as a CDM candidate if $M_{1} \gtrsim 200 \mathrm{GeV}$. This hypothesis is not compatible with the observed Baryon asymmetry if the gauginos or further sleptons other than the sneutrino are still present in the plasma at temperatures below $T_{C}$ or if the masses associated to the bino and wino bear the same sign. On the other hand, a scenario with opposite sign gaugino mass parameters and both gauginos sufficiently heavy $M_{1}, M_{2} \gtrsim 500 \mathrm{GeV}$ (and slepton masses bigger than $T_{C}$ ) is compatible with the sneutrino CDM hypothesis.

\section{Acknowledgments}

We thank D. Semikoz and U. Sarkar for helpful discussions. St. K. would like to 
thank the Institute for Nuclear Research in Moscow for hospitality and the Deutscher Akademischer Austauschdienst for financial support.

\section{References}

[1] Y. Fukuda et al., the Super-Kamiokande Collaboration, Phys. Lett. B 467 (1999) 185.

[2] S.M. Bilenky, C. Giunti and W. Grimus, Prog. Part. Nucl. Phys. 43 (1999) 1 and hep-ph/9812360.

[3] H.V. Klapdor-Kleingrothaus in Proc. Int. Conf. on Lepton- and Baryon-Number Non-Conservation, Trento, Italy, April 20-25, 1998, IOP, Bristol (1999); H.V. Klapdor-Kleingrothaus, Int. J. Mod. Phys. A 13 (1998) 3953.

[4] M. Hirsch, H.V. Klapdor-Kleingrothaus and S. Kovalenko, Phys. Lett. B 398 (1997) 311.

[5] H.E. Haber and G.L. Kane, Phys. Rep. 117 (1985) 76.

[6] Y. Grossmann and H.E. Haber, Phys. Rev. Lett. 78 (1997) 3438.

[7] B.A. Campbell, S. Davidson and K.A. Olive, Nucl. Phys. B 399 (1993) 111; M. Plümacher, Nucl. Phys. B 530 (1998) 207; L. Covi, E. Roulet and F. Vissani, Phys. Lett. B 384 (1996) 169.

[8] M. Hirsch, H.V. Klapdor-Kleingrothaus, St. Kolb and S. Kovalenko, Phys. Rev. D 57 (1998) 2020.

[9] M. Hirsch, H.V. Klapdor-Kleingrothaus and S. Kovalenko, Phys. Rev. D 57 (1998) 1947.

[10] L.J. Hall, T. Moroi and H. Murayama, Phys. Lett. B 424 (1998) 305.

[11] V.A. Kuzmin, V.A. Rubakov and M. Shaposhnikov, Phys. Lett. B 155 (1985) 36.

[12] E.W. Kolb and M.S. Turner, The Early Universe (Addison-Wesley, Redwood City, CA, 1990).

[13] U. Sarkar, Phys. Lett. B 390 (1997) 97.

[14] K. Kajantie, M. Laine, K. Rummukainen and M. Shaposhnikov, Nucl. Phys. B 466 (1996) 189. 
[15] V.A. Rubakov and M.E. Shaposhnikov, Usp. Fiz. Nauk 166 (1996) 493, Phys. Usp. 39 (1996) 461; A. Riotto, hep-ph/9807454

[16] G.D. Moore, Phys. Rev. D 59 (1999) 014503.

[17] L. Baudis et al., Heidelberg-Moscow Collab., Phys. Rev. Lett. 83 (1999) 41.

[18] St. Kolb, M. Hirsch, H.V. Klapdor-Kleingrothaus and O. Panella, Phys. Lett. B 478 (2000) 262. 